\title{
Beyond current research practice: methodological considerations in MS rehabilitation research (Is designing the perfect rehabilitation trial the Holy Grail or a Gordian knot?)
}

Rehabilitation is an essential aspect of symptomatic and supportive treatment for people with multiple sclerosis (MS). MS rehabilitation research has grown considerably, but the volume of robust evidence to guide effective clinical practice in the form of high quality randomised controlled trials (RCTs) is low ${ }^{1}$. Such robust research evidence is important because it is a key driver for optimising patient outcomes and service delivery ${ }^{2}$, and influencing health policy decision making.

This discussion paper explores some of the challenges of undertaking robust clinical trials within this field. We acknowledge the importance of using a variety of research methods to fully understand whether and how rehabilitation interventions work, but focus on RCTs since they are arguably regarded as a superior research design to determine whether a treatment is beneficial ${ }^{3}$. However, RCTs have largely been modelled on pharmacological trials where the precise dose of the intervention, double blind procedures, and safety reporting mechanisms can be strictly adhered to. Such issues become much more complex in rehabilitation trials. Therefore, additional guidelines for conducting and reporting non-pharmacological trials have been published ${ }^{4}$. Despite these advances, reporting has not yet significantly improved ${ }^{5}$ and specific challenges persist.

We describe some key methodological issues faced in rehabilitation trials, and draw upon examples from both within and outside the MS field, where these dilemmas have been (at least partially) tackled. We make some suggestions as to how future research can be scientifically robust whilst remaining relevant and "fit for purpose" in generating new knowledge, which can meaningfully shape rehabilitation practice and improve patient outcomes.

\section{Trial designs - efficacy vs. effectiveness trials}

All RCT designs share some common features (e.g., randomisation), but they also differ based on the general aims of the RCT. Efficacy (or explanatory) trials, which are often highly controlled, aim to answer whether an intervention produces benefits for patients under ideal (often laboratory) conditions, and are typically used to demonstrate 'proof-of-principle' for newer interventions. An example of an efficacy rehabilitation trial is Dalgas et al. ${ }^{5}$. 
Effectiveness (or pragmatic) trials aim to answer whether an intervention produces benefits for patients under usual clinical (real-world) conditions, to determine whether the intervention translates to clinical practice, and how and with whom the intervention could be used $^{6}$. An example of an effectiveness rehabilitation trial is Boesen et al. ${ }^{7}$. Despite this distinction, efficacy and effectiveness trials should be seen as two ends of a continuum ${ }^{8}$. Both serve different functions ${ }^{9}$, can be equally robust, and are required. The key, at the design stage, is determining what kind of trial is needed, with clarity and transparency about the research questions being posed. Based on this distinction, trials vary in relation to Participants, Intervention, Control, and Outcomes (PICO).

\section{Participants}

\section{(i) Selection}

Several factors influence participant recruitment, including whether the study is an efficacy trial (requiring a homogenous sample meeting specific entry criteria) or an effectiveness trial (requiring a more heterogeneous sample to enable generalisability). Trials are sometimes criticised for not recruiting participants akin to patients seen in clinical practice (with multiple comorbidities and illness profiles, from different backgrounds), or are drawn from specific services or geographical locations with regional idiosyncrasies, thereby questioning the generalisability of the findings ${ }^{10}$. This criticism can be addressed if trialists specify the trial type. Also important is distinguishing between the proportion of participants eligible for the intervention and those unable to participate in the research study (exclusions such as reduced capacity for consent, enrolment on another trial), to provide a clearer indication of how many people would benefit from the intervention if it were part of routine practice.

Participants should be recruited based on the goal of the rehabilitation intervention. Some MS rehabilitation trials have not done this. For instance, a Cochrane review of exercise interventions for fatigue in MS concluded that most trials did not explicitly include people who experienced fatigue ${ }^{11}$. This could have affected the overall 'effectiveness' of the intervention.

Often neglected in terms of participant selection is a participant's readiness for the intervention or behavioural change. This is particularly important in rehabilitation trials where participants need to actively engage in the treatment to derive benefit. Using strategies such as motivational interviewing has demonstrated improvement in participant engagement, 
therapy adherence, outcomes, and patient-provider relationships ${ }^{12}$. Consideration of this variable as an inclusion criterion or moderator may enhance understanding about differential treatment effects.

As per CONSORT guidelines for non-pharmacological trials ${ }^{13}$, a table showing baseline demographic and clinical characteristics for each group should be presented. Related to this, as recommended by an international workshop addressing comorbidity in clinical trial design and conduct ${ }^{14}$, trialists should present comorbidity status of participants, and where possible, evaluate treatment response, tolerability, and safety based on comorbidity. Furthermore, when applicable, a description of care providers (case volume, qualification, expertise, etc.) and rehabilitation context should also be documented.

\section{(ii) Sample size}

In RCTs a sample with the specific attribute of interest is selected and studied, and the findings "extrapolated"15. Having a small study risks making a Type II error. Indeed, most trials with 'negative results' are with small samples, under-powered to detect significant differences between groups ${ }^{16}$. Having 'too many' people also poses ethical and resource issues $^{15}$. Therefore, an a priori sample size calculation is required to identify the numbers needed. However, we still find MS rehabilitation trials where sample size calculations are not reported.

Publication bias (i.e., only trials with positive findings being reported ${ }^{17}$ in meta-analysis are more likely to affect small studies ${ }^{18}$. Given that most MS rehabilitation trials are 'small', it is concerning that many of the systematic reviews (including several Cochrane Reviews) have not formally assessed publication bias, despite evidence that publication bias exists in some MS rehabilitation trials ${ }^{11,19}$. Assessing this will enable a fuller understanding of the state of the science.

There are, however, complexities in determining sample size in trials of 'complex interventions', such as rehabilitation, because determining a clinically meaningful difference on outcome measures can be difficult (see below).

\section{Intervention}


Rehabilitation is a "process of assessment, treatment and management by which the individual (and their family/carers) are supported to achieve their maximum potential for physical, cognitive, social and psychological function, participation in society and quality of living” (p2, $\left.{ }^{20}\right)$. It is often provided by different healthcare professionals, based on specific needs (such as neuropsychological rehabilitation), or specific symptoms (such as mobility problems), and in a holistic fashion by a coordinated inter-disciplinary team ${ }^{21}$, with access to several specialist health professions ${ }^{22}$.

\section{(i) Dose of treatment}

Unlike pharmacological interventions, within much of MS rehabilitation research the optimal 'dose' of the intervention, and when to deliver this (e.g., time post-relapse) is yet to be established. The reasons for this are manifold. In some areas (e.g., cognitive rehabilitation), few large, well-conducted trials are available, so dose-response analyses have not been performed. Where rehabilitation packages containing several 'modules' are evaluated, oftentimes, it is the whole package that is evaluated, so the relative effect of each module is unknown. Also, the complex nature of the intervention means that combined effects of a combination of modules may be greater than the sum of the parts. Finally, there is a recognition, in other areas of neurology, that higher doses of rehabilitation training do not always provide better results ${ }^{23}$. This may also be the case in MS, for instance when impairments (such as fatigue or weakness) and disabilities are very severe. Rule-based dosefinding trial designs are one way of tackling this issue. More commonly used in pharmaceutical research, they have recently been undertaken in stroke rehabilitation to determine the optimal dose for specific intervention prior to undertaking an efficacy study ${ }^{24}$. These studies utilise an adaptive design approach to enable dose escalation/de-escalation according to pre-set rules and a mathematical sequence, thereby allowing exploration of the dose-response relationship, whilst minimising sample size and maintaining participant safety.

\section{(ii) Description of the intervention}

Describing and characterising the "black box" of rehabilitation is important to enable replication of studies and implementation of interventions based on what was trialled. Checklists and guidelines like the Template for Intervention Description and Replication $\left(\right.$ TIDieR) ${ }^{25}$ can help researchers report interventions more thoroughly, however this is not yet commonplace in MS rehabilitation research. For instance, in a systematic review of trials evaluating cognitive rehabilitation $(\mathrm{k}=52)$, the reporting of key aspects of the intervention 
was judged to be poor, particularly in relation to content of the intervention, delivery mode, and proposed mechanism of action ${ }^{26}$.

\section{(iii) Active ingredients}

What constitutes the active ingredients of rehabilitation, which typically uses a multicomponent biopsychosocial model ${ }^{27,28}$, is difficult to determine. For instance, many interventions incorporate several settings, healthcare providers and treatment techniques based on the premise that a successful outcome is a result of this cross-fertilisation of inputs. Further, the core principle of many rehabilitation interventions is to actively engage individuals in change processes such as learning, practicing, and developing skills to enhance coping and adaptation across diverse outcomes.

Psychotherapy research demonstrates that where different therapies produce similar outcomes, this is because of a set of 'common factors', with 'therapeutic alliance' between the patient and therapist being most relevant ${ }^{29}$. MS rehabilitation research has rarely evaluated such common factors, however, there is some consensus regarding what the 'key' (if not 'active') ingredients, and mode of delivery are in some rehabilitation interventions (e.g., falls ${ }^{30,31}$, fatigue $\left.{ }^{32-34}\right)$.

Understanding the mechanism of action, its target, and desired effect is also important in defining the active ingredients. Advances in neuroscience, such as functional imaging techniques that enable investigation of neuroplastic adaptations ${ }^{35}$, provide important insights into this. But equally, easy-to-collect patient-reported outcomes can improve our understanding of underlying mechanisms ${ }^{36}$. While these may not be a trial's main outcomes, embedding this work within clinical trials provides opportunities to advance knowledge of how treatments work, and to develop new recovery-oriented strategies in MS. For instance, video recordings of therapists delivering the trial intervention can enable us to determine the degree to which therapist competence is associated with patient outcomes ${ }^{37}$, or mixed methods designs collecting patient and therapist perspectives can improve our understanding of the perceived effects and experiences of interventions ${ }^{38}$.

\section{(iv) Fidelity of intervention delivery}

Given the complex nature of most rehabilitation interventions, in the context of a trial, it is vital for reliability and validity of the findings that the intervention is delivered as intended, 
consistently across participants and sites, and any deviations from the planned intervention recorded. While some MS trials describe intervention fidelity ${ }^{39,40}$, this is not yet the norm, despite guidelines available on treatment fidelity in health behaviour change studies ${ }^{41}$, which can be adapted for MS rehabilitation trials. This said, assessing fidelity in complex interventions is challenging, because it not only requires an examination of what was delivered (content), but also how the intervention was delivered (process). Much of the literature around fidelity assessments in trials tends to focus on 'implementation fidelity' (i.e., how the intervention was delivered, dose received, etc.) and 'theoretical fidelity' (i.e., how the intervention delivered matched the theoretical underpinning of the intervention $)^{42}$. However, these still neglect the quality of the intervention delivery and the quality of the therapeutic relationship. This, however, is rarely assessed in MS rehabilitation trials.

\section{(v) Targeting of interventions}

Related to the notion of 'person-centredness', a criticism levelled against RCTs in rehabilitation is that oftentimes the intervention is not sufficiently individualised. This is particularly challenging with group-based interventions, although some attempts have been made to personalise interventions. For instance, in a memory rehabilitation RCT, baseline memory assessment scores were used to highlight which memory strategies might be more useful than others, and homework exercises enabled participants to test memory strategies within their home settings to determine what adaptations needed to be made to the strategies $^{43}$. Similar approaches have been used in MS RCTs of fatigue ${ }^{36}$ and falls ${ }^{44}$ interventions.

\section{(vi) Treatment adherence}

The success of a treatment is partly dependent on patients' adherence. Where patients are required to actively participate over a long period, there is often considerable variation in adherence ${ }^{45}$. While there are some good examples of MS rehabilitation trials that report adherence ${ }^{46}$, this appears less well described in some domains (such as memory/cognition ${ }^{47}$ ). It would be beneficial for trials to report the minimum number of sessions (or other marker of dose) required to demonstrate effect, and the actual number of sessions that participants engage in. The risk of not specifying the actual 'amount' of intervention participants received is that where trials show 'no intervention effect', it is difficult to disentangle whether the treatment was ineffective or whether people did not engage in the required dose of the intervention. 
Despite some challenges in their use ${ }^{48}$, wearable technologies (sensors and/or software applications on watches, smartphones, shoes, etc.) can be embedded within RCTs, and can provide an opportunity to objectively capture adherence or behavioural changes outside clinical settings, within the 'real world'.

\section{Control groups}

There are several approaches available when deciding which control condition is most appropriate $^{49}$. Whilst one option is to withhold active treatment, ethical issues need to be considered, such as the nature of the target problem, the state of science with regard to the effectiveness of existing treatments, and lack of acceptability to participants - which might affect recruitment, retention and data integrity. An alternative is the wait-list control design, which has been used in several RCTs of multi-disciplinary packages of rehabilitation ${ }^{50}$ and single component interventions such as tele-rehabilitation ${ }^{51,52}$. This approach can control for the effects of time, guaranteeing eventual intervention, reflecting the realities of clinical waiting lists, and being more acceptable to potential participants. However, there is the risk that simply knowing that they will eventually receive the intervention may change the nature of the controls, and may not represent the experience or response to those who do not receive an intervention. Controls that involve comparison to usual care are more commonly used in rehabilitation RCTs, and whilst useful for examining the added value of an intervention, they are complicated by the fact that usual care is often highly variable and may require large samples to achieve adequate statistical power.

Active controls, wherein active treatments are compared to the test intervention (e.g., equivalence trials), are challenging in terms of defining the active ingredients, and being clear about what elements are most essential to control for (e.g., dose, attention, etc.) in behavioural-based 'complex' interventions, with multiple 'active ingredients' that may be difficult to characterise. Nevertheless, this has been achieved in some rehabilitation trials of exercise $^{53,54}$ and cognition ${ }^{55}$, although these trials have investigated single interventions that typically have not incorporated a behavioural approach. Finally, some trials have used attention placebo controls, wherein control participants receive an intervention that is plausible, mimicking the amount of time and attention received by the treatment group, but is thought not to have a specific effect on the outcomes of interest. Arguably, this can be ethically defensible when no effective treatment has proven to exist, which is commonplace 
for MS rehabilitation, and when participants know what to expect in each treatment arm. This is a challenge for behavioural-based interventions since it requires double blinding, which often cannot be accomplished in the MS rehabilitation field. Studies in MS rehabilitation have achieved this, but have involved "passive" interventions not requiring active engagement by the participants to affect target outcomes ${ }^{56,57}$. Others have attempted to include attention placebo groups, where the facilitators do not present the presumed active ingredients in the sessions, but do not (and cannot) prevent participants from discussing the issues they struggle with (e.g., memory ${ }^{55}$ ).

\section{Outcomes}

\section{(i) Choice of outcomes}

The International Classification of Functioning, Disability and Health (ICF) is the World Health Organisation's "framework for measuring health and disability at both individual and population levels" $" 58$ and is consistent with the aims of rehabilitation. Outcomes can be at an impairment, activity, or participation level, but we cannot assume that improvements at impairment level will generalise to reductions in activity limitations and participation restrictions. There are however, examples where this is the case, for example strengthening of lower limbs has shown to improve mobility in people with $\mathrm{MS}^{59}$. Whether or not translation from impairment to activity limitations/participation is seen, should be explicitly and systematically reported so that clear conclusions can be drawn.

It is important to consider health economic outcomes, particularly in pragmatic trials where policy decisions need to be based on both clinical and cost-effectiveness data. MS rehabilitation trials have begun addressing this ${ }^{43,44,60-63}$. Quality of life (QoL) is sometimes considered a desirable 'general' outcome of rehabilitation trials. While this is laudable, there are challenges in using such an outcome. As a primary outcome for specific types of rehabilitation (e.g., cognitive rehabilitation), improvements may not be observed on QoL measures as a consequence of the intervention because QoL is often a composite construct (e.g., measures may include items related to fatigue, pain, social and physical function), whereas the intervention may only be able to change one aspect (e.g., cognition or social function). Baumstarck et al. ${ }^{64}$ provide a good summary of some of the issues in assessing QoL in people with MS. Similar issues may arise when using generic activities of daily living scales or participation scales wherein a total score is derived from multiple items. In such instances, subset or domain-specific scores may be more appropriate as a primary outcome. 
Capturing adverse events in complex intervention trials is challenging, because the chance of detecting them may differ between treatment arms. Furthermore, in some rehabilitation trials, the primary outcome may also be considered as an adverse event (e.g., falls frequency in falls prevention interventions ${ }^{65,66}$. Despite these challenges, and although many rehabilitation trials are considered 'low risk', it is important to consider the potential adverse events, how these will be assessed, monitored, reported, and addressed ${ }^{60,61,67}$.

\section{(ii) Number of outcomes}

It is tempting to use a large number of outcomes to capture any potential effect in rehabilitation trials. This has the disadvantage of increasing the risk of false positive findings. Nonetheless, in a complex intervention trial, it may be appropriate to have different types of outcomes that relate to the key clinical or economic variables. In addition, mechanistic measures may be useful to unpack why an intervention worked (or not).

\section{(iii) Defining a primary outcome}

Scientific rigor in RCTs rightly demands that the primary and secondary outcomes are defined beforehand, because the trial is powered based on finding a clinically relevant effect of the intervention on the primary outcome. All outcomes need to be described as primary or secondary (in the protocol or trial registry), and should be analysed and reported, in line with CONSORT guidelines ${ }^{13}$. Unfortunately, reviews find that not all MS rehabilitation trials do this ${ }^{11,19}$. Selecting and reporting on a limited number of pre-specified (primary) outcomes, with a clear rationale for selecting them, limits the risk of bias and is an important consideration to building a solid evidence base for rehabilitation interventions. Core Outcome Sets for rehabilitation trials in MS have been recommended for various types of rehabilitation (e.g., exercise interventions ${ }^{68}$ ), and the recommendations of the American Physical Therapy Association's Multiple Sclerosis Outcome Measures Task Force ${ }^{69}$ can be used to guide trialists in selecting outcome measures.

\section{(iv) Timing of outcomes}

The time point of primary concern should be specified in advance of the analysis. Because it is likely that the best outcome is seen immediately after the intervention, outcomes assessed at this time-point can be useful to determine effectiveness of the intervention. However, this leaves uncertainty as to the long-term benefits. Treatment effects can gradually wear off ${ }^{34}$ or, 
particularly with regard to impact on activities and participation, can take longer to establish $^{70}$. The selection of one time point (either long- or short-term) limits insight into what happens during the rehabilitation process, and restricts the use of powerful longitudinal analysis techniques that can help to understand the course of treatment effects. The time point of primary concern, however, should be specified in advance of the analysis.

\section{(v) Defining a clinically important/meaningful difference}

Labelling the result of a trial as "positive" or "negative" is often inappropriately based on whether or not statistical significance is achieved for the primary outcome $e^{71-73}$. It is more important to show that a pre-specified difference in scores between groups is clinically relevant ${ }^{74}$. Nevertheless, sound evidence on minimal clinically important differences (MCID) of outcomes is often unavailable, barring some good exceptions ${ }^{43,75-78}$.

\section{(vi) Statistical analysis}

Several MS rehabilitation trials conduct significance testing comparing baseline with followup outcome scores (within group changes) rather than comparing randomised groups directly (between-group differences), which can be highly misleading ${ }^{79}$. Analyses plans should be decided before the data are locked and deviations from this plan should be specified in the publication of trial results, with some arguing that pre-specified statistical analysis plans should be published prospectively ${ }^{80}$.

\section{A summary of these issues and suggestions is provided in Table 1.}

[Insert Table 1 here]

\section{Conclusions and future directions}

We have made much progress in undertaking, conducting, and reporting RCTs in MS rehabilitation research, but there is some way to go yet.

We believe we can improve the design, conduct, and reporting of trials by following internationally-accepted RCT guidelines (see www.equator-network.org), and by developing a critical mass of MS rehabilitation researchers, perhaps by extending and intensifying cooperation within organisations such as Rehabilitation in MS (www.eurims.org), to 
undertake high quality rehabilitation trials. We may need to extend our collaborations with "critical friends" outside of the area of MS to facilitate inter- and cross-disciplinary ways of addressing shared methodological dilemmas. One such group might be the newly formed Cochrane Rehabilitation Field (www.rehabilitation.cochrane.org), which may be a vehicle to improve research methods (for trials and syntheses), publicise results, and drive forward evidence-based clinical care.

The ICF is an excellent framework to map rehabilitation trial outcomes. More, however, can be made in relation to the 'personal' and 'environmental' aspects of the ICF, perhaps as understanding prognostic factors that relate to outcome, or in relation to clinical implementation of positive trials.

We also believe work is needed to ensure that journal editors and reviewers judge rehabilitation trials in the light of the specific challenges posed in designing these trials, and view the merits of the trials based on internationally-recognised RCT guidelines for complex intervention trials, rather than compare them with pharmacological trials. We need research that covers the full cycle of the Medical Research Council's framework for the development and evaluation of complex interventions ${ }^{81}$ - from developing and modelling interventions, to feasibility and pilot testing, to pragmatic trials and implementation studies. Most MS rehabilitation trials have focussed on the earlier parts of the cycle, and there is a need for more pragmatic trials evaluating clinical and cost-effectiveness, for translation of findings from research to clinical practice. 'Null' or 'negative' results should be published. Some journals, including MSJ, accept "short reports on null or negative results". This is important for the advancement of science. We would, however, suggest that such trials should not be limited to "short" reports, and indeed, may be worthy of longer reports given that such results need to be expanded further and the pathways for future research in the field should be clearly stated. Such papers have spurred great developments in the field ${ }^{82}$.

We need to continue to critique, challenge and develop RCT designs. Because most rehabilitation interventions are complex interventions, outcomes-focussed trials only address whether an intervention is effective or not and do not offer answers to more nuanced questions: "When and for what kind of patient is this intervention effective?" 
It is clear that undertaking scientifically rigorous research that is clinically meaningful is a complex problem, but we believe it is not unsolvable. Just as Alexander the Great disentangled the Gordian knot, so too can clinicians, researchers and people with MS work together to systematically conquer the methodological conundrums that currently challenge us.

\section{References}

1. Haselkorn JK, Hughes C, Rae-Grant A, et al. Summary of comprehensive systematic review: rehabilitation in multiple sclerosis: report of the Guideline Development, Dissemination, and Implementation Subcommittee of the American Academy of Neurology. Neurology. 2015; 85: 1896-903.

2. Emparanza JI, Cabello JB and Burls AJ. Does evidence-based practice improve patient outcomes? An analysis of a natural experiment in a Spanish hospital. Journal of Evaluation in Clinical Practice. 2015; 21: 1059-65.

3. Bothwell LE, Greene JA, Podolsky SH and Jones DS. Assessing the gold standardlessons from the history of RCTs. New England Journal of Medicine. 2016; 374: 2175-81.

4. Boutron I, Altman DG, Moher D, Schulz KF and Ravaud P. CONSORT statement for randomized trials of nonpharmacologic treatments: a 2017 update and a CONSORT extension for nonpharmacologic trial abstracts. Annals of Internal Medicine. 2017; 167: 40-7. 5. Dalgas U, Stenager E, Jakobsen J, et al. Resistance training improves muscle strength and functional capacity in multiple sclerosis. Neurology. 2009; 73: 1478-84.

6. Ware JH and Hamel MB. Pragmatic trials - guides to better patient care. New England Journal of Medicine. 2011; 364: 1685-7.

7. Boesen F, Norgaard M, Trenel P, et al. Longer term effectiveness of inpatient multidisciplinary rehabilitation on health-related quality of life in MS patients: a pragmatic randomized controlled trial - The Danish MS Hospitals Rehabilitation Study. Multiple sclerosis (Houndmills, Basingstoke, England). 2018; 24: 340-9.

8. Loudon K, Treweek S, Sullivan F, Donnan P, Thorpe KE and Zwarenstein M. The PRECIS-2 tool: designing trials that are fit for purpose. British Medical Journal. 2015; 350: h2147.

9. Lurie JD and Morgan TS. Pros and cons of pragmatic clinical trials. Journal of Comparative Effectiveness Research. 2013; 2: 53-8.

10. Kennedy-Martin T, Curtis S, Faries D, Robinson S and Johnston J. A literature review on the representativeness of randomized controlled trial samples and implications for the external validity of trial results. Trials. 2015; 16: 495.

11. Heine M, van de Port I, Rietberg MB, van Wegen EE and Kwakkel G. Exercise therapy for fatigue in multiple sclerosis. The Cochrane Library. 2015: Art. No.: CD009956.

12. Medley AR and Powell T. Motivational Interviewing to promote self-awareness and engagement in rehabilitation following acquired brain injury: A conceptual review.

Neuropsychological Rehabilitation. 2010; 20: 481-508.

13. Boutron I, Altman DG, Moher D, Schulz KF, Ravaud P and Group ftCN. CONSORT Statement for Randomized Trials of Nonpharmacologic Treatments: A 2017 Update and a CONSORT Extension for Nonpharmacologic Trial AbstractsCONSORT Nonpharmacologic Treatments. Annals of Internal Medicine. 2017; 167: 40-7.

14. Marrie RA, Miller A, Sormani MP, et al. The challenge of comorbidity in clinical trials for multiple sclerosis. Neurology. 2016; 86: 1437-45. 
15. Nayak BK. Understanding the relevance of sample size calculation. Indian Journal of Ophthalmology. 2010; 58: 469.

16. Moher D, Dulberg CS and Wells GA. Statistical power, sample size, and their reporting in randomized controlled trials. JAMA. 1994; 272: 122-4.

17. Egger $\mathrm{M}$ and Smith GD. Bias in location and selection of studies. British Medical Journal. 1998; 316: 61.

18. Sterne JA, Gavaghan D and Egger M. Publication and related bias in meta-analysis: power of statistical tests and prevalence in the literature. Journal of Clinical Epidemiology. 2000; 53: 1119-29.

19. Rosti-Otajärvi EM and Hämäläinen PI. Neuropsychological rehabilitation for multiple sclerosis. Cochrane Database of Systematic Reviews. 2014: Art. No.: CD009131.

20. Turner-Stokes L. Specialist neuro-rehabilitation services: providing for patients with complex rehabilitation needs. British Society of Rehabilitation Medicine, 2010.

21. Choi BC and Pak AW. Multidisciplinarity, interdisciplinarity and transdisciplinarity in health research, services, education and policy: 1 . Definitions, objectives, and evidence of effectiveness. Clinical and Investigative Medicine. 2006; 29: 351.

22. Croft A, Mynors G, Roberts M, Doncaster D, Bowen A and Trust M. MS Forward View: a consensus for the future of MS services. Multiple Sclerosis Trust. 2016.

23. Lang CE, Lohse KR and Birkenmeier RL. Dose and timing in neurorehabilitation: prescribing motor therapy after stroke. Current opinion in neurology. 2015; 28: 549-55.

24. Colucci E, Clark A, Lang CE and Pomeroy VM. A rule-based, dose-finding design for use in stroke rehabilitation research: methodological development. Physiotherapy. 2017; 103: 414-22.

25. Hoffmann TC, Glasziou PP, Boutron I, et al. Better reporting of interventions: template for intervention description and replication (TIDieR) checklist and guide. British Medical Journal. 2014; 348: g1687.

26. Mhizha-Murira JR, Drummond A, Klein OA and dasNair R. Reporting interventions in trials evaluating cognitive rehabilitation in people with multiple sclerosis: a systematic review. Clinical rehabilitation. 2018; 32: 243-54.

27. Engel GL. The Need for a New Medical Model: A Challenge for Biomedicine. Holistic Medicine. 1989; 4: 37-53.

28. Wade DT and Halligan PW. The biopsychosocial model of illness: a model whose time has come. Clinical rehabilitation. 2017; 31: 995-1004.

29. Martin DJ, Garske JP and Davis MK. Relation of the therapeutic alliance with outcome and other variables: a meta-analytic review. Journal of Consulting and Clinical Psychology. 2000; 68: 438.

30. Finlayson M, Cattaneo D, Cameron M, et al. Applying the RE-AIM framework to inform the development of a multiple sclerosis falls-prevention intervention. International Journal of MS Care. 2014; 16: 192-7.

31. Gunn H, Endacott R, Haas B, Marsden J and Freeman J. Development of a balance, safe mobility and falls management programme for people with multiple sclerosis. Disability and Rehabilitation. 2018; 40: 2857-66.

32. Beckerman H, Blikman LJ, Heine M, et al. The effectiveness of aerobic training, cognitive behavioural therapy, and energy conservation management in treating MS-related fatigue: the design of the TREFAMS-ACE programme. Trials. 2013; 14: 250.

33. Blikman LJ, van Meeteren J, Twisk JW, et al. Effectiveness of energy conservation management on fatigue and participation in multiple sclerosis: A randomized controlled trial. Multiple Sclerosis Journal. 2017; 23: 1527-41. 
34. van den Akker LE, Beckerman H, Collette EH, et al. Cognitive behavioral therapy positively affects fatigue in patients with multiple sclerosis: Results of a randomized controlled trial. Multiple Sclerosis Journal. 2017; 23: 1542-53.

35. Lipp I and Tomassini V. Neuroplasticity and Motor Rehabilitation in Multiple Sclerosis. Frontiers in Neurology. 2015; 6.

36. van den Akker L, Beckerman H, Collette E, et al. Cognitive behavioural therapy for MS-related fatigue explained: A longitudinal mediation analysis. Journal of Psychosomatic Research. 2018; 106: 13-24.

37. Lincoln NB, das Nair R, Bradshaw L, et al. Cognitive Rehabilitation for Attention and Memory in people with Multiple Sclerosis: study protocol for a randomised controlled trial (CRAMMS). Health Technology Assessment. in press.

38. Bakker M, Schipper K, Koopman FS, Nollet F and Abma TA. Experiences and perspectives of patients with post-polio syndrome and therapists with exercise and cognitive behavioural therapy. BMC Neurology. 2016; 16: 23.

39. Hayes S, Uszynski MK, Motl RW, et al. Randomised controlled pilot trial of an exercise plus behaviour change intervention in people with multiple sclerosis: the Step it Up study. BMJ Open. 2017; 7: e016336.

40. Morrison JD, Becker H and Stuifbergen AK. Evaluation of Intervention Fidelity in a Multisite Clinical Trial in Persons With Multiple Sclerosis. Journal of Neuroscience Nursing. 2017; 49: 344-8.

41. Bellg AJ, Borrelli B, Resnick B, et al. Enhancing treatment fidelity in health behavior change studies: best practices and recommendations from the NIH Behavior Change Consortium. Health Psychology. 2004; 23: 443.

42. Haynes A, Brennan S, Redman S, Williamson A, Gallego G and Butow P. Figuring out fidelity: a worked example of the methods used to identify, critique and revise the essential elements of a contextualised intervention in health policy agencies. Implementation Science. 2015; 11: 23.

43. Lincoln NB, das Nair R, Bradshaw L, et al. Cognitive Rehabilitation for Attention and Memory in people with Multiple Sclerosis: study protocol for a randomised controlled trial (CRAMMS). Trials. 2015; 16: 556.

44. Gunn H, Andrade J, Paul L, et al. Balance Right in Multiple Sclerosis (BRiMS): a guided self-management programme to reduce falls and improve quality of life, balance and mobility in people with secondary progressive multiple sclerosis: a protocol for a feasibility randomised controlled trial. Pilot and feasibility studies. 2017; 4: 26.

45. Romberg A, Virtanen A, Ruutiainen J, et al. Effects of a 6-month exercise program on patients with multiple sclerosis. Neurology. 2004; 63: 2034.

46. Khan F, Pallant JF, Brand C and Kilpatrick TJ. Effectiveness of rehabilitation intervention in persons with multiple sclerosis: a randomised controlled trial. Journal of neurology, neurosurgery, and psychiatry. 2008; 79: 1230-5.

47. das Nair R, Martin KJ and Lincoln NB. Memory rehabilitation for people with multiple sclerosis. Cochrane Database Syst Rev. 2016; 3: CD008754.

48. Izmailova ES, Wagner JA and Perakslis ED. Wearable Devices in Clinical Trials: Hype and Hypothesis. Clinical Pharmacology and Therapeutics. 2018; 104: 42-52.

49. Brigham GS, Feaster DJ, Wakim PG and Dempsey CL. Choosing a control group in effectiveness trials of behavioral drug abuse treatments. Journal of Substance Abuse Treatment. 2009; 37: 388-97.

50. Freeman JA, Langdon DW, Hobart JC and Thompson AJ. The impact of inpatient rehabilitation on progressive multiple sclerosis. Annals of neurology. 1997; 42: 236-44. 
51. Tallner A, Streber R, Hentschke C, et al. Internet-Supported Physical Exercise Training for Persons with Multiple Sclerosis-A Randomised, Controlled Study. International Journal of Molecular Sciences. 2016; 17: 1667.

52. Pilutti LA, Dlugonski D, Sandroff BM, Klaren R and Motl RW. Randomized controlled trial of a behavioral intervention targeting symptoms and physical activity in multiple sclerosis. Multiple Sclerosis. 2013; 20: 594-601.

53. Wens I, Dalgas U, Vandenabeele F, et al. High Intensity Exercise in Multiple Sclerosis: Effects on Muscle Contractile Characteristics and Exercise Capacity, a Randomised Controlled Trial. PloS one. 2015; 10: e0133697.

54. Zimmer P, Bloch W, Schenk A, et al. High-intensity interval exercise improves cognitive performance and reduces matrix metalloproteinases-2 serum levels in persons with multiple sclerosis: A randomized controlled trial. Multiple Sclerosis. 2018; 24: 1635-44. 55. das Nair R and Lincoln NB. Evaluation of rehabilitation of memory in neurological disabilities (ReMiND): a randomized controlled trial. Clin Rehabil. 2012; 26: 894-903. 56. Nielsen JF, Sinkjaer T and Jakobsen J. Treatment of spasticity with repetitive magnetic stimulation; a double-blind placebo-controlled study. Multiple Sclerosis. 1996; 2: 227-32.

57. Richards TL, Lappin MS, Acosta-Urquidi J, et al. Double-blind study of pulsing magnetic field effects on multiple sclerosis. Journal of Alternative and Complementary Medicine. 1997; 3: 21-9.

58. Organisation WH. International classification of functioning, disability, and health : ICF. Version 1.0. Geneva : World Health Organization, 2001.

59. Latimer-Cheung AE, Pilutti LA, Hicks AL, et al. Effects of exercise training on fitness, mobility, fatigue, and health-related quality of life among adults with multiple sclerosis: a systematic review to inform guideline development. Archives of physical medicine and rehabilitation. 2013; 94: 1800-28.e3.

60. Humphreys I, Drummond AE, Phillips C and Lincoln NB. Cost-effectiveness of an adjustment group for people with multiple sclerosis and low mood: a randomized trial. Clinical Rehabilitation. 2013; 27: 963-71.

61. Thomas S, Thomas PW, Kersten P, et al. A pragmatic parallel arm multi-centre randomised controlled trial to assess the effectiveness and cost-effectiveness of a group-based fatigue management programme (FACETS) for people with multiple sclerosis. Journal of neurology, neurosurgery, and psychiatry. 2013; 84: 1092-9.

62. Tosh J, Dixon S, Carter A, et al. Cost effectiveness of a pragmatic exercise intervention (EXIMS) for people with multiple sclerosis: economic evaluation of a randomised controlled trial. Multiple Sclerosis. 2014; 20: 1123-30.

63. Freeman JA HW, Jarrett L, Hawton A, Barton A, Dennett R, Jones BG, Zajicek J, Creanor S. Evaluation of a home-based standing frame programme in people with progressive Multiple Sclerosis (SUMS): a pragmatic, multi-centre, randomised, controlled trial and cost-effectiveness analysis. Lancet Neurology in press.

64. Baumstarck K, Boyer L, Boucekine M, Michel P, Pelletier J and Auquier P. Measuring the Quality of Life in Patients with Multiple Sclerosis in Clinical Practice: A Necessary Challenge. Multiple Sclerosis International. 2013; 2013: 8.

65. Cattaneo D, Rasova K, Gervasoni E, Dobrovodska G, Montesano A and Jonsdottir J. Falls prevention and balance rehabilitation in multiple sclerosis: a bi-centre randomised controlled trial. Disability and Rehabilitation. 2016; 40: 522-6.

66. Gunn H and Freeman J. Repeated falls: A key outcome or an adverse event? Physiotherapy Research International. 2012; 17: 1-3. 
67. Ioannidis JA, Evans SW, Gøtzsche PC and et al. Better reporting of harms in randomized trials: An extension of the consort statement. Annals of Internal Medicine. 2004; 141: 781-8.

68. Paul L, Coote S, Crosbie J, et al. Core outcome measures for exercise studies in people with multiple sclerosis: recommendations from a multidisciplinary consensus meeting. Multiple sclerosis (Houndmills, Basingstoke, England). 2014; 20: 1641-50.

69. Cohen ET, Potter K, Allen DD, et al. Selecting Rehabilitation Outcome Measures for People with Multiple Sclerosis. International journal of MS care. 2015; 17: 181-9.

70. Freeman JA, Langdon DW, Hobart JC and Thompson AJ. Inpatient rehabilitation in multiple sclerosis: do the benefits carry over into the community? Neurology. 1999; 52: 50-6.

71. Chavalarias D, Wallach JD, Li AH and Ioannidis JP. Evolution of Reporting P Values in the Biomedical Literature, 1990-2015. JAMA. 2016; 315: 1141-8.

72. Gardner MJ and Altman DG. Confidence intervals rather than P values: estimation rather than hypothesis testing. British Medical Journal. 1986; 292: 746-50.

73. van Tulder M, Malmivaara A, Hayden J and Koes B. Statistical significance versus clinical importance: trials on exercise therapy for chronic low back pain as example. Spine. 2007; 32: 1785-90.

74. Heine M, Verschuren O, Hoogervorst EL, et al. Does aerobic training alleviate fatigue and improve societal participation in patients with multiple sclerosis? A randomized controlled trial. Multiple Sclerosis Journal. 2017; 23: 1517-26.

75. Baert I, Smedal T, Kalron A, et al. Responsiveness and meaningful improvement of mobility measures following MS rehabilitation. Neurology. 2018; 91: e1880-e92.

76. de Groot V, Beckerman H, Uitdehaag BM, et al. The usefulness of evaluative outcome measures in patients with multiple sclerosis. Brain : a journal of neurology. 2006; 129: 2648-59.

77. Mehta L, McNeill M, Hobart J, et al. Identifying an important change estimate for the Multiple Sclerosis Walking Scale-12 (MSWS-12v1) for interpreting clinical trial results. Multiple Sclerosis Journal 2015; 1: 2055217315596993-.

78. Widener GL and Allen DD. Measurement characteristics and clinical utility of the 29item Multiple Sclerosis Impact Scale. Archives of physical medicine and rehabilitation. 2014; 95: 593-4.

79. Bland JM and Altman DG. Comparisons against baseline within randomised groups are often used and can be highly misleading. Trials. 2011; 12: 264.

80. Finfer $\mathrm{S}$ and Bellomo R. Why publish statistical analysis plans? Critical Care and Resuscitation. 2009; 11: 5-6.

81. Craig P, Dieppe P, Macintyre S, Michie S, Nazareth I and Petticrew M. Developing and evaluating complex interventions: the new Medical Research Council guidance. British Medical Journal. 2008; 337: a1655.

82. Eyssen IC, Steultjens MP, de Groot V, et al. A cluster randomised controlled trial on the efficacy of client-centred occupational therapy in multiple sclerosis: good process, poor outcome. Disability and rehabilitation. 2013; 35: 1636-46. 